\title{
1 3-dimensional spatially organized PEG-based hydrogels for an aortic valve co-culture model
}

2 Daniel S. Puperi ${ }^{\dagger, a}$, Liezl R. Balaoing ${ }^{\dagger, a}$, Ronan W. O’Connell ${ }^{\mathrm{b}}$, Jennifer L. West ${ }^{\mathrm{c}}$, K. Jane Grande-Allen ${ }^{*}$,

3 a. Department of Bioengineering, Rice University, Houston, TX 77005

4 b. Department of Biomedical Engineering, University of Glasgow, Glasgow, United Kingdom

5 c. Department of Biomedical Engineering, Duke University, Durham, NC 27708

$6 \uparrow$ Authors contributed equally to this work

$7 \quad *$ Corresponding author

8

9 Address for correspondence:

10 K. Jane Grande-Allen, Ph. D.

11 Department of Bioengineering

12 Rice University

13 P.O. Box 1892, MS 142

14 Houston, TX 77251-1892, USA

15 Tel: +1 7133483704

16 Fax: +1 7133485877

17 Email: grande@ rice.edu 
Abstract

Physiologically relevant in vitro models are needed to study disease progression and to develop

21 and screen potential therapeutic interventions for disease. Heart valve disease, in particular, has no early

22 intervention or non-invasive treatment because there is a lack of understanding the cellular mechanisms

23 which lead to disease. Here, we establish a novel, customizable synthetic hydrogel platform that can be

24 used to study cell-cell interactions and the factors which contribute to valve disease. Spatially localized

25 cell adhesive ligands bound in the scaffold promote cell growth and organization of valve interstitial cells

26 and valve endothelial cells in 3D co-culture. Both cell types maintained phenotypes, homeostatic

27 functions, and produced zonally localized extracellular matrix. This model extends the capabilities of in

28 vitro research by providing a platform to perform direct contact co-culture with cells in their

29 physiologically relevant spatial arrangement.

30

31

32

33

34 Keywords: heart valve, co-culture, hydrogel, scaffold, endothelialisation, biomimetic material

35

36

37

38

39

40 


\section{Introduction}

Over the past 50 years, in vitro study of cell behavior has been important for understanding biological processes. Cells are studied with in vitro models before attempting to study them in the in vivo context because in vitro research is less expensive, is higher throughput, and offers better control of culture conditions. Much in vitro research has historically been performed in 2D, but 3D cell culture has become an increasingly important technique as research has demonstrated that many cell types retain their

47 physiological behavior in 3D culture because of the relevant spatial presentation of microenvironmental cues[1]. Most 3D cell culture research, however, starts with cells and/or scaffolding in a homogeneous state, which does not recapitulate the physiological arrangement of tissue. Advancements in 3D culture systems, such as designed scaffold heterogeneity and co- or tri-culture of multiple cell types have given researchers greater flexibility and greater ability to mimic the natural microenvironment. Recent research has demonstrated that a heterogeneous 3D culture scaffold can be constructed using additive manufacturing[2,3], photopatterning[4], and/or layering techniques[5,6]. Co- and tri- culture approaches are of interest because they allow researchers to study cell-cell interactions in a controlled environment[7] and have been particularly useful in studying angiogenesis in multiple tissue contexts, including 56 cancer[8]. vitro models has proven challenging[9]. Difficulties in optimizing culture conditions and maintaining 59 long-term 3D co-culture without phenotypical change represent notable concerns[7], especially when attempting to understand the complex processes which cause the onset and progression of age-correlated 61 diseases.

To address the need for an improved in vitro co-culture model, we have designed a synthetic hydrogel platform for studying cell-cell interactions in their natural spatial arrangement. This tunable model provides control over parameters such as cell-type specific ligand presentation and mechanical properties, thereby more accurately mimicking natural ECM presentation to the cells. We used this system to develop a co-culture model in which we can study the factors which contribute to heart valve 

the two cell types interact. Valvular interstitial cells (VICs) are fibroblast-like cells that occupy the interior of the valve and are responsible for ECM maintenance [10]. The valvular endothelial cells

70

71 (VECs) line the exterior surfaces of valves and regulate tissue inflammatory and thrombotic responses, as well as signal underlying VICs and remodel valve ECM $[11,12]$. Both cell types have been implicated in valve disease; understanding how the cells contribute to the disease state may help the development of a non-invasive treatment option. Co-culture models have been used to investigate VIC-VEC interactions[13-15], but this model is the first to facilitate cell specific binding to the scaffold through use of zonally organized ECM ligand presentation. The model was developed to expand on previous studies by investigating some additional questions about valve cell interactions, including how VECs respond to the presence of VICs in the scaffold and which external cues from the ECM and surroundings are necessary to maintain valve homeostasis. The strategy of presenting a physiologically relevant 3D spatial arrangement of cell-specific cues can be applied to other endothelialized or epithelialized tissues which have a similar stratified arrangement of cells and matrix components. This model is the next step toward creating an in vitro 3D co-culture platform for high throughput analysis of disease development and drug screening.

\section{Materials and Methods}

\section{Valve cell isolation and culture}

Aortic valve leaflets were dissected from fresh young adult (3-6 month old) porcine hearts acquired from a commercial abattoir (Fisher Ham and Meats, Spring TX). Aortic valve endothelial cells (VECs) and valve interstitial cells (VICs) were harvested following previously described methods [11,1618]. Dissected aortic valve leaflets were enzymatically digested in a collagenase II/dispase solution for 1 hour at $37^{\circ} \mathrm{C}$. Next, VECs on the surface of the leaflets were isolated via gentle scraping using sterile swabs. The VECs were cultured on tissue culture plastic (TCPS) coated with $2.5 \%$ gelatin in EGM-2 medium and growth factor bullet kit (Lonza, Walkerville, MD) with $2 \%$ fetal bovine serum (FBS) and $1 \%$ 
penicillin/streptomycin/amphotericin in an incubator $\left(37^{\circ} \mathrm{C}, 5 \% \mathrm{CO}_{2}, 95 \%\right.$ humidity $)$. At first cell passage, CD31 positive VECs were purified using anti-CD31 (Millipore, Billerica, MA) antibodyconjugated CELLection magnetic sorting beads (Invitrogen, Carlsbad, CA). If VECs were used after freeze, a second bead sort purification was performed. VECs were used between passages 2 to 5 .

VICs were harvested by mincing aortic valve leaflets that had been denuded of their endothelium. The minced leaflets were digested in a collagenase III buffer for 4 hours. The cells were isolated by straining the digested tissue solution through a 70 micron cell strainer and then seeded onto TCPS flasks. VICs were cultured in a standard humidified incubator at $37^{\circ} \mathrm{C}$ with 5\% $\mathrm{CO}_{2}$ in 50:50 DMEM:F12 media (Corning, Tewksbury, MA) with 1\% FBS (Lonza) and 1\% penicillin/streptomycin/amphotericin (Lonza) and buffered with 1\% 1M 4-(2-hydroxyethyl)-1-piperazineethanesulfonic acid (HEPES, Thermo Fisher Scientific, Waltham, MA). VICs were used between passages 1 to 3 .

Porcine aortic endothelial cells (AECs) were harvested from fresh porcine aortic tissues (Fisher Ham and Meats) following the same methods as VEC isolation, and cultured using the same culture conditions and medium as VECs. AECs were also purified at passage 1 for CD31 positive expression using magnetic bead sorting. AECs were used between passages 2 to 5 .

Flow cytometry for CD31 expression using mouse anti-porcine CD31 antibody (AbD serotec, Oxford, United Kingdom) was performed to verify endothelial cell phenotype. Culture media for all cell cultures were changed every 2-3 days. All cell cultures in subsequent hydrogel scaffold based experiments used a 50:50 ratio of VIC:VEC culture medium based on the above formulations.

\section{Peptides}

MMP-2 and 9 sensitive peptide sequence GGGPQG $\downarrow$ IWGQGK (hereafter identified as PQ) was synthesized using solid phase synthesis (APEX 396, Aapptec, Louisville, KY). Fibronectin-derived integrin binding peptide sequence RGDS and laminin-derived syndecan binding peptide CRKRLQVQLSIRT (hereafter identified as RKR) were purchased from American Peptide Company (Vista, CA). RKR was dissolved in DMSO at a concentration of $15 \mathrm{mM}$ and stored at $-80^{\circ} \mathrm{C}$ in aliquots. 
PEG-RGDS and PEG-PQ-PEG was synthesized as previously described [19]. $3.4 \mathrm{kDa}$ monoacrylated PEG succinimidyl valerate (Laysan Bio, Arab, AL) was reacted at a molar ratio of 1.2:1 RGDS: PEG-SVA or 1:2.1 PQ: PEG-SVA overnight in HEPBS buffer (Santa Cruz Biotechnology, Dallas, TX) at $\mathrm{pH}$ 8.0. The PEGylated peptide solution was dialyzed against pure water in a 3500 125 molecular weight cutoff dialysis membrane (SpectrumLabs, Rancho Dominguez, CA) for 3 days to 126 remove unreacted precursors. After dialysis, the solutions were sterile filtered and kept sterile throughout 127 freezing, lyophilization, and experimental use. Gel permeation chromatography was used to confirm PEG-peptide conjugation and proton NMR was used to ensure the acrylate groups remained intact.

\section{Co-culture construction}

The hydrogel-based endothelialized aortic valve model (HEAVM) was constructed over 2 days as depicted in Figure 1A. First a $500 \mu \mathrm{m}$ thick, $12 \mu \mathrm{m}$ diameter base gel of 10\% w/v 6 kDA PEGDA was made in a silicone mold. PEGDA was synthesized from 6kDa PEG (Sigma) using acryloyl chloride be able to manipulate the cell-encapsulated HEAVM gel without direct contact thereby preventing 136 damage. The base gel was blotted dry using sterilized Kimwipes and a $250 \mu \mathrm{m}$ thick square of PDMS 137 with a $5 \mathrm{~mm}$ diameter cut out was placed on the base gel to be used as a mold for the HEAVM gel. VICs 138 were released from a TCPS flask using 0.25\% Trypsin with EDTA (Corning), diluted in media, and 139 counted. Six gels were made simultaneously to minimize the time that VICs were in the photoinitiator 140 solution. 900,000 VICs were centrifuged at 750x g and resuspended in $60 \mu \mathrm{L}$ of $4 \%$ w/v PEG-PQ-PEG 141 and $2 \mathrm{mM}$ PEG-RGDS photoinitiator solution $\left(15 \times 10^{6}\right.$ cells $\left./ \mathrm{ml}\right)$. An established white light photoinitiator 142 system[21] consisting of $1.5 \%$ triethanolamine (TEOA), $10 \mu \mathrm{M}$ Eosin Y, and $0.35 \%$ (v/v) 1-vinyl-2-

143 pyrillidinone (NVP) in HEPES buffered saline was used to crosslink hydrogels. $9 \mu \mathrm{L}$ of the cell 144 suspension was pipetted into each PDMS mold and photo-crosslinked under 160 kLux of white light 

mold was removed, and VIC encapsulated gels were put in an incubator overnight in VIC media to wash out unreacted photoinitiator and allow VICs to grow in the scaffold.

After overnight incubation, the VIC encapsulated gels were washed in sterile PBS and blotted dry with sterilized Kimwipes. The $15 \mathrm{mM}$ RKR in DMSO was diluted to $3 \mathrm{mM}$ using Eosin Y photoinitiator solution. $10 \mu \mathrm{L}$ of the $3 \mathrm{mM}$ RKR solution was pipetted onto the top of the hydrogel. The gels were placed under white light for 1 minute to initiate a free-radical thiol-ene reaction between the cysteine of the RKR peptide and unreacted vinyl groups on the surface of the VIC encapsulated hydrogel. The gels were then washed extensively to remove unbound RKR peptide. Sterile PBS was pipetted onto the surface of the hydrogel to physically dislodge unbound RKR and then the gels were placed in VIC media in an incubator for 10 minutes to allow for diffusion of unbound RKR and photoinitiator solution. This process was repeated $3 x$ to ensure only covalently bound RKR was left on the surface while keeping the encapsulated VICs viable. VECs were released from TCPS flasks using 0.25\% Trypsin with EDTA (Corning), counted, and resuspended in VEC media at a concentration of 200,000 cells/ml. Cloning columns $(10 \mathrm{~mm} \times 10 \mathrm{~mm})$ were used to restrict VEC seeding to the HEAVM surface. The cloning columns were placed on top of the base gel around the HEAVM scaffold, creating a sealed volume in which $150 \mu \mathrm{L}$ of VEC cell suspension was pipetted to seed 30,000 cells per gel. The cloning columns concentrated the VECs in the vicinity of the HEAVM scaffold and prevented VECs from attaching to the bottom of the well plate. The outside of the cloning columns was flooded with 50:50 VIC:VEC media and the cloning columns were removed after 1 hour in culture. The HEAVM gels were cultured for 7 days. In experiments where VIC-only and VEC-only controls were compared to the HEAVM coculture, the same procedure was followed. VIC-only scaffolds were constructed by encapsulating VICs in 4\% PEG-PQ-PEG hydrogels with $2 \mathrm{mM}$ PEG-RGDS. The gels were incubated in VIC media overnight and the next day RKR was crosslinked to the surface, but no VECs were seeded onto the gels. VEC-only scaffolds were constructed by forming acellular 4\% PEG-PQ-PEG hydrogels with 2 mM PEG-RGDS, 
170 incubating them in VIC media overnight, and crosslinking RKR peptide to the surface followed by

171 seeding VECs on the surface as detailed above.

172

\section{$173 \quad$ Fluorescently Labeled Peptides}

succinimidyl ester, respectively, following the manufacturer's protocol (Invitrogen, Carlsbad, CA).PEG-

176 RGDS was dissolved at $20 \mathrm{mg} / \mathrm{mL}$ in $0.1 \mathrm{M}$ sodium carbonate buffer and RKR was first dissolved in

177 DMSO at $30 \mathrm{mM}$ and then adjusted to $10 \mathrm{mg} / \mathrm{ml}$ in $0.1 \mathrm{M}$ sodium carbonate buffer. The AlexaFluor

178 succinimidyl ester was dissolved at $10 \mathrm{mg} / \mathrm{mL}$, added dropwise to the peptide solutions, and vortexed

179 lightly for 1 hour. The solution was dialyzed against pure water and lyophilized before use. PEG-RGDS-

180 AF488 was incorporated into a 4\% PEG-PQ-PEG hydrogel and RKR-AF532 was covalently bound on

181 top of the hydrogel in the same manner as described above for unlabeled peptides. $500 \mu \mathrm{m}$ thick sections

182 were sliced with a razor blade and laid on their side to image the cross-section of the hydrogel using a

183 confocal microscope (LSM 510 Live 5, Zeiss, Oberkochen, Germany)

Mechanical characterization of hydrogels

HEAVM scaffold and base gel stiffness were characterized through compression testing.

187 Hydrogels consisting of 4\% PEG-PQ-PEG with $2 \mathrm{mM}$ PEG-RGDS were crosslinked under white light in 188 cylindrical PDMS molds $6 \mathrm{~mm}$ in diameter by $0.8 \mathrm{~mm}$ thick for 35 seconds. The hydrogels were soaked 189 in PBS overnight and subject to unconfined compression at a rate of $0.02 \mathrm{~mm} / \mathrm{sec}$ using a Bose

190 Electroforce ELF 3200 mechanical testing machine equipped with a $1000 \mathrm{~g}$ load cell (Bose Electroforce,

191 Eden Prairie, MN). After this non-destructive testing, RKR was added to the surface of each gel as

192 described in the previous section and crosslinked under white light for 1 minute. Unreacted RKR was

193 washed off and the gels were left to soak in PBS overnight. Hydrogels were mechanically tested again

194 using the same parameters to determine how the bulk stiffness of the hydrogel changed with additional

195 crosslinking. For all tests, the compressive modulus was calculated as the least-squares linear fit slope of 
the stress/strain curve between $5 \%$ and $15 \%$ strain. Volumetric swelling was calculated by using cross-

197 sectional area and thickness measurements from stereoscope images.

$2 D$ cell adhesion and phenotype verification

To verify the ability of valve cells to adhere to adhesive peptides RKR and RGDS, VECs and

VICs were seeded onto the surface of PEG-PQ-PEG scaffolds that had either: $3 \mathrm{mM}$ of RKR conjugated

202 only on the hydrogel surface, 2 mM PEG-RGDS conjugated throughout the bulk hydrogel, or combined

203 RKR (on the surface)-RGDS (bulk) conjugated hydrogels. AECs were used as a reference endothelial

204 population, and were also seeded onto all hydrogel scaffold combinations. After 7 days of culture, the

205 cells were imaged using brightfield microscopy to visualize cell morphology, and then immunostained for

206 CD31 and $\alpha$ SMA.

\section{Cytotoxicity assessment}

The ability of the cells to survive the encapsulation and seeding process was assessed using the

210 Live/Dead viability kit (Invitrogen, Carlsbad, CA) following manufacturer's instructions. After 3 days in

211 culture, HEAVM constructs were incubated in $2 \mu \mathrm{M}$ of calcein AM and $4 \mu \mathrm{M}$ of ethidium homodimer-1

212 in culture media for 30 minutes at room temperature. The gels were washed once in PBS and immediately

213 imaged with confocal microscopy (A1-Rsi, Nikon, Tokyo, Japan). Cells were counted using the ImageJ

214 3D Objects Counter plug-in.

215

\section{Immunohistochemistry}

Cells cultured on all hydrogel scaffold combinations were fixed in $4 \%$ paraformaldehyde for 45 minutes and permeabilized with $0.25 \%$ Triton-X for 15 minutes at RT. Next, samples were the blocked with PBS with $3.5 \%$ bovine serum albumin (BSA) at $4{ }^{\circ} \mathrm{C}$ overnight. The samples were incubated with

220 primary antibodies of interest at manufacturer recommended dilutions in PBS and $0.35 \%$ BSA overnight

221 at $4{ }^{\circ} \mathrm{C}$. Negative controls were left incubating in PBS with $0.35 \%$ BSA. The next day, samples were 
222 incubated with fluorescent secondary antibodies (AlexaFluor 488/555/633; Pacific orange, Invitrogen)

223 overnight at $4^{\circ} \mathrm{C}$. After washing, samples were counterstained with DAPI and phalloidin for 1 hour prior 224 to imaging with confocal microscopy (Nikon A1-Rsi or Zeiss LSM 510).

Antigens investigated in this study included the endothelial marker CD31 (Abcam, Cambridge,

227 BD610296, 1:100); and extracellular proteins laminin (Lam, Abcam, ab14055, 1:200), collagen type IV

228 (Col IV, Abcam ab6586, 1:500), perlecan (Pln, Abcam, ab26265, 1:1000), collagen type I (Col I, Abcam, $229 \mathrm{ab34710,1:50)}$ and fibronectin (FN, abcam, ab6328, 1:100).

\section{Scanning Electron Microscopy}

on the surface of HEAVM scaffolds that had been in culture for 28 days and to visualize adhered serial ethanol gradient (10-100\%) and dried in a critical point dryer (EMS 850, Electron Microscopy back from one HEAVM sample to visualize the underlying VICs. The dried samples were sputter coated with $10 \mathrm{~nm}$ of gold (Desk V, Denton Vacuum, Moorestown, NJ) and imaged using an FEI Quanta 400 ESEM microscope (FEI, Hillsboro, OR) in high vacuum mode at $15 \mathrm{kEV}$.

\section{Quantitative RT-PCR}

Quantitative RT-PCR (qPCR) was used to quantify the relative gene expression of $\alpha$ SMA, CD31 and eNOS between all 3D scaffold culture groups ( $n=4-6)$. After 7 days in culture, cell seeded hydrogels

244 were homogenized and mRNA was extracted and purified using Quick-RNA MiniPrep Kit (Zymo

245 Research, Irvine, CA). The purified mRNA was reverse-transcribed into cDNA ( $1^{\text {st }}$ Strand cDNA 246 synthesis kit, Takara Bio, Otsu, Japan). All cDNA samples were stored at $-20^{\circ} \mathrm{C}$ until use. qPCR was 247 performed using QuantiTect SYBR Green PCR Master Mix (Clontech Laboratories, Mountain View, CA) 

were calculated using the REST 2009 program[22] using the pairwise fixed reallocation randomization test for statistical significance (p-value less than 0.05). Relative gene expression of aSMA was normalized to VIC-only seeded scaffolds; and CD31 and eNOS gene expression were normalized to

252 VECs cultured on TCPS. All primers used (Integrated DNA Technologies, Coralville, Iowa) are listed in 253 table S1.

Because the HEAVM scaffold contained 2 different cell types, the $\mathrm{C}_{\mathrm{t}}$ values of the housekeeping 255 gene, GAPDH, were adjusted based on the average number of VICs and VECs in the scaffold. This 256 adjustment was performed to portray the behavior of the cells of interest more accurately (VICs for scaffolds $(\mathrm{n}=5)$ giving a ratio of $37.3 \%$ VECs and $62.7 \%$ VICs. Based on the GAPDH primer efficiency of $94.2 \%$, the HEAVM GAPDH $\mathrm{C}_{\mathrm{t}}$ value was reduced by 0.72 for $\alpha$ SMA and 1.22 for eNOS and CD31.

\section{Nitric Oxide Production}

A total nitric oxide (NO) detection kit (Enzo Life Sciences, Farmingdale, NY) was used to measure the sum of nitrites $\left(\mathrm{NO}_{2}{ }^{-}\right)$and nitrates $\left(\mathrm{NO}_{3}{ }^{-}\right)$in collected media samples to determine the levels of nitric oxide produced by HEAVM co-cultures. Because nitric oxide is quickly degraded into nitrates and nitrites in culture, the kit coverts the collected NO breakdown products into nitrites that can be colorimetrically detected when reacted with Greiss reagents.

Fresh culture media samples were collected from HEAVM co-culture, VEC-only, and VIC-only scaffolds after 3 days since last medium change, on day 5 of culture (n=5). Fresh 50:50 VIC-VEC culture medium was used as a baseline control. Following the kit protocol, media samples were diluted 1:2 with

270 NO kit reaction buffer and ultrafiltered through a 10,000 molecular weight cut off filter (Millipore). Next,

$27150 \mu \mathrm{L}$ of each sample was incubated in a microtiter plate with NADH and nitrate reductase enzyme for 30

272 min. at $37^{\circ} \mathrm{C}$. The mixtures were then reacted with Greiss reagents for $10 \mathrm{~min}$. at room temp. The peak 273 absorbance of each of the solutions were measured between 400-600nm with a spectrophotometer (Cary 
275 was calculated using a standard curve produced using serial dilutions of a nitrate standard. The nitrite

276 concentration from the fresh 50:50 culture medium controls were used as a baseline and subtracted from

277 the nitrite concentrations measured in the cell seeded hydrogel scaffolds.

Platelet adhesion assay

A quantitative platelet adhesion assay adapted from Xu et al. was performed to assess the

281 HEAVM co-cultures' ability to prevent platelet activation and adhesion [23]. Whole blood was collected

282 from consented healthy adults using a Rice University IRB approved protocol and centrifuged to isolate

283 platelet rich plasma (PRP). The PRP was then treated with prostacyclin $(5 \mu \mathrm{g} / \mathrm{mL}$ of PRP), washed $2 \mathrm{x}$

284 with CGS buffer (12.9 mM sodium citrate/33.33 mM glucose/123.2 mM NaCl, pH 7) to remove

285 remaining plasma. The platelets were resuspended in calcium free Tyrode's buffer at half the original

286 volume of collected PRP and were dyed with Sudan B Black solution (5\% w/v in 70\% ethanol) for 2

287 hours at RT. After staining, the platelets were washed 3x with PBS to remove excess dye, counted using a

288 hemocytometer, and resuspended to a concentration of $10 \times 10^{6}$ platelets $/ \mathrm{mL}$ in PBS. Next, $5 \times 10^{6}$ platelets

289 were added to HEAVM co-culture, VEC-only, VIC-only, and blank hydrogel scaffolds and incubated for

2901 hour in a shaking incubator at $37^{\circ} \mathrm{C}$. All constructs were then washed $3 \mathrm{x}$ with PBS to remove unbound

291 platelets. Next, each scaffold was carefully removed from the PEG base gel and placed in a

292 microcentrifuge tube. DMSO was added to each sample to lyse the bound platelets and release the SBB

293 dye into solution. Afterwards equal volume of PBS was added to the lysed platelet suspension in order to

294 normalize to the PBS measured in the standard curve. The solutions for each sample were collected into

295 cuvettes and the peak absorbance were measured at $614 \mathrm{~nm}$ using a Cary $60 \mathrm{UV}$-Vis spectrophotometer.

296 The total adhered platelets were calculated based on a standard curve made from serial dilutions of the

297 dyed platelet stock solution, and then normalized to the amount of adhered platelets on the blank

298 scaffolds. 

were fixed in 4\% PFA for 30 minutes. Gels were washed 3x in PBS and counter stained with DAPI for 1 hour before being imaged on Nikon A1-Rsi confocal microscope. Each of the fixed samples were incubated with $15 \mu \mathrm{g} / \mathrm{mL}$ of fluorescent acetylated low-density lipoprotein complexes (Dil-AcLDL, Invitrogen) 4 hours prior to the addition of the platelets. Dil-AcLDL is commonly used to identify endothelial cells in culture by their ability to take up the fluorescent AcLDL complexes. Fluorescent and brightfield images were overlaid to identify areas of the hydrogels where activated platelets adhered.

\section{Results}

Constructed hydrogel-based aortic valve model scaffolds have relevant spatial arrangement of cell adhesive peptides

HEAVM scaffolds were constructed over a 2 day period (Figure 1A). A $12 \mathrm{~mm}$ diameter by 0.5 mm thick $10 \%(\mathrm{w} / \mathrm{v}) 6 \mathrm{kDa}$ PEGDA base gel was used to facilitate moving the HEAVM scaffold with tools without damage. The stiffness of the base gel was $126.7 \pm 2.5 \mathrm{kPa}$ with a volumetric swelling ratio of $27.5 \pm 2.5 \%$. The $5 \mathrm{~mm}$ diameter by $250 \mu \mathrm{m}$ thick VIC encapsulated PEG-PQ-PEG gel was seeded with VECs on the surface. The resulting co-culture system consisted of a VEC covered-VIC encapsulated scaffold on top of the PEGDA base gel (Figure 1B). 1C). PEG-RGDS+AlexaFluor 488 was incorporated into the bulk of the PEG-PQ-PEG gel (Figures 1C-2 and 1C-4) while RKR+AlexaFluor 532 was crosslinked to the surface (Figure 1C-1). Some excitement of the AF488 fluorophore due to the $532 \mathrm{~nm}$ laser was observed, but there was a distinct difference between the fluorescent signal on the surface with RKR (Figures 1C-1 and 1C-2) compared to the surface without RKR (Figure 1C-3 and 1C-4).

The volumetric swelling ratio of $22.9 \pm 1.5 \%$ was calculated by comparing the initially cross-

323 linked volume to the fully swollen volume of the hydrogel. The bulk compressive modulus of the swollen 324 hydrogels was $5.95 \pm 0.20 \mathrm{kPa}$. RKR was cross-linked to the surface of the hydrogels after compression 
testing and then left overnight in PBS. There was no significant difference in swelling after this secondary crosslinking $(\mathrm{p}=0.61)$, but the compressive modulus increased to $10.25 \pm 0.98 \mathrm{kPa}(\mathrm{p}=0.02)$ because unreacted free-acrylate groups in the bulk of the hydrogel crosslinked to completion.

\section{Combined RKR-RGDS conjugated scaffolds support VEC monolayer formation}

VECs, VICs, and aortic endothelial cells (AECs) were seeded onto PEG-PQ-PEG scaffolds with RGDS and RKR ligands to observe the cell adhesion behavior. VECs adhered to the surface of RGDS only, RKR only, and combined RGDS-RKR scaffolds, and maintained characteristic cobblestone morphology as well as positive CD31 expression in all conditions (Figure 2, middle row). There was no positive VEC expression of $\alpha$ SMA on any of the scaffold combinations. However, VECs did not form continuous monolayers on the RGDS-only gels, whereas VECs on the RKR-only and the RGDS-RKR scaffolds formed continuous, tightly packed monolayers. No VEC invasion into the MMP degradable scaffold was observed in any of the conditions.

AECs served as reference endothelial cell population. Similar to VECs, AECs adhered onto the surface of all ligand combinations and maintained their cobblestone morphology and positive CD31 expression (Figure 2, top row). No $\alpha$ SMA expression was observed in any AEC culture. However, AECs seeded on RGDS only and RKR only did not form continuous monolayers on the scaffold surface. In contrast, AECs on the RGDS-RKR scaffold formed continuous, tightly packed endothelial monolayers. 2D cultures of VICs adhered and spread onto both RGDS-only and RGDS-RKR conjugated scaffolds. VICs adhered to RKR only scaffolds, but aggregated with surrounding VICs rather than spreading on the surface or invading into the hydrogel (Figure 2, bottom row). VICs cultured in 2D atop the RGDS-only and RGDS-RKR conditions invaded into the hydrogel scaffold and stained strongly for aSMA.

HEAVM co-cultures maintain valve cell phenotypes and physiologically relevant localization 

(88\%) seeded on the surface remained viable (Supplemental figure S1). Encapsulated VICs showed lower 352 survival with $66 \%$ of cells staining positive for calcein.

After 7 days in culture, immunohistological stains of HEAVM co-cultures showed that VECs localized on the surface formed a monolayer and were positive for CD31, while VICs within the hydrogel had low, diffuse expression of activation marker $\alpha$ SMA (Figure 3A). SEM pictographs of the HEAVM 356 confirmed the formation of a tight, continuous VEC monolayer on the scaffold surface (Supplemental 357 figures S2A and S2B). SEM and confocal imaging demonstrated healthy 3-dimensional spreading by 358 VICs within the hydrogel scaffold (Supplemental figure S2C-D). VEC-only surface-seeded scaffolds stained positive for CD31 and negative for $\alpha$ SMA, and did not invade into the PQ-degradable hydrogel. 3D encapsulated VIC-only scaffolds stained negative for CD31, but stained positive for $\alpha$ SMA when localized closer to the bottom of the scaffold (Figure S3). expression versus VIC-only encapsulated scaffolds ( $p<0.05$ ). VICs grown on TCPS had significantly higher $\alpha$ SMA mRNA expression than all other groups ( $\mathrm{p}<0.05)$ (Figure 3B). VIC-only scaffolds had 1000x less CD31 mRNA expression than the baseline VEC TCPS group (p<0.05) (Figure 3C). HEAVM and VEC-only scaffolds had no significant differences in CD31 gene expression relative to VEC TCPS. The GAPDH $\mathrm{C}_{\mathrm{t}}$ value for the HEAVM scaffold was adjusted in order to account for only the cell of interest (VICs for $\alpha$ SMA and VECs for CD31) as described in the materials and methods. Adjusting the value did not change the results or statistical significance (Figures S4A, S4B).

HEAVM co-cultures stained for endothelial nitric oxide synthase (eNOS) co-localized with

374 CD31-positive VECs at the surface of the scaffold co-culture (Figure 4A). HEAVM co-culture ( $\mathrm{p}=0.14)$ 375 and VEC-only ( $\mathrm{p}=0.06)$ scaffolds expressed similar levels of eNOS mRNA as VECs on TCPS (Figure 
4B). VIC-only scaffolds expressed significantly lower (1000x) levels of eNOS mRNA than all other groups $(\mathrm{p}<0.05)$. The GAPDH $\mathrm{C}_{\mathrm{t}}$ value for the HEAVM scaffold eNOS expression was adjusted to account for VECs only as described in the materials and methods. If GAPDH from both VICs and VECs in the HEAVM scaffold was used in the analysis, qPCR results indicate that the HEAVM expressed less eNOS mRNA ( $\mathrm{p}=0.049)$ than the VEC-only case (Figure S4C).

NO byproducts released into culture medium were measured to quantify the levels of nitric oxide (NO) produced by the cells (Figure 4C). Less than $1 \mu \mathrm{M}$ of nitrite was measured in VIC-only scaffolds. The VEC-only and HEAVM scaffolds had significantly higher nitrite concentrations than the VIC-only scaffolds $(\mathrm{p}<0.05)$.

\section{VEC monolayer reduces platelet adhesion to underlying HEAVM scaffold}

Platelet adhesion on the hydrogel constructs was quantified through the measured absorbance of lysed adhered platelets. VEC-only scaffolds had $0.33 \%$ less adhered platelets $(\mathrm{p}=0.26)$ than acellular, blank hydrogels, while HEAVM scaffolds had two times more adhered platelets $(p=0.06)$ than blank (Figure 5A). HEAVM co-cultures had significantly greater platelet adhesion than VEC-only scaffolds $(\mathrm{p}<0.005)$. VIC-only scaffolds promoted significantly greater platelet adhesion than all other groups, including 10x more adhered platelets than found on VEC-only scaffolds $(\mathrm{p}<0.005)$.

VEC-only scaffolds had a continuous endothelial monolayer that stained positively for dilAcLDL with minimal platelet adhesion (Figure 5B, Supplemental figure S5A). Large platelet clumps adhered to the VIC-only scaffolds, especially in areas where VICs were visible on the surface of the hydrogel (Figure 5C, Supplemental figure S5B). Acellular blank gels had regions of adhered platelet clumps scattered throughout the hydrogel surface (Figure 5D, Supplemental figure S5C). The majority of HEAVM co-culture scaffolds had a dil-AcLDL positive endothelial monolayer free from adhered platelets on the scaffold surface (Figure 5E). However, platelet clumps could be seen on small regions on the HEAVM scaffold with dil-AcLDL negative cells, blank regions, and areas where there were disruptions in the endothelial monolayer (Figure 5F-G, Supplemental figure S5D). 
Long term HEAVM co-cultures produce zonally organized extracellular matrix

HEAVM co-cultures cultured for 28 days produced an abundance of basement membrane proteins laminin, perlecan, and collagen type IV on the surface of hydrogel scaffold (Figure 6A). Collagen type I fibers were present throughout the interior of the hydrogels and fibronectin, a ubiquitous 407 ECM protein, was present both on the surface and throughout the HEAVM scaffold (Figure 6B).

\section{Discussion}

In vitro culture of primary cells is an important technique for learning how cells behave in the

411 body and how they respond to both normal and pathological stimuli. However, the utility of in vitro

412 culture is limited by the inability to mimic the natural microenvironment perfectly. Advancements in cell

413 culture technology improve on shortcomings by providing physiologically relevant scaffolds and cues in

414 platforms which can then be used to study disease states, response to external stimuli, and/or preliminary 415 drug-screening. In this study, we present a hydrogel-based endothelialized aortic valve model (HEAVM)

416 construct to co-culture aortic valve cells, VICs and VECs. This platform was designed to allow for precise 417 control and independent modulation of various factors that can influence cell behavior, including 418 mechanical properties and cell ligand concentration and arrangement. PEG has been widely used as a 419 scaffold material due to its innate property to resist non-specific protein adsorption and its tunability that 420 allow researchers to tailor material properties and incorporate covalently bound cell signaling molecules 421 through different crosslinking mechanisms [24]. Previous studies have successfully supported VIC 422 viability and function in long-term 3D culture using PEG hydrogels with PQ MMP degradable peptide 423 sequences and RGDS adhesion ligands [25,26]. VEC cultures, on the other hand, have been shown to 424 maintain healthy phenotype and monolayer formation when cultured on RKR peptide-functionalized 425 PEGDA hydrogels, but lose stability and hemostatic balance when cultured on RGDS only-functionalized 426 hydrogels[27].Thus, the HEAVM platform was constructed by combining the strategies to encapsulate 427 VICs in 3D throughout the interior of PEG-PQ-PEG/PEG-RGDS hydrogel and to functionalize the 
surface with RKR adhesive ligands to support VEC monolayer growth (Figure 1). This design mimics the natural arrangement of heart valves with VICs throughout the valve interior and VECs lining the bloodcontacting surfaces $[28,29]$. VECs seeded in 2D onto the RKR functionalized surfaces (both RKR only and the RKR-RGDS combination) quickly formed stable monolayers and did not invade into the MMP-2 degradable gel (Figure 2). These results are in agreement with published data which has demonstrated the synergistic effects of integrin and syndecan in the binding of endothelial cells to their substrate [30].

434 Conversely, VICs did not spread when cultured in 2D on top of RKR only hydrogels, but retained cell 435 spreading and proliferation when cultured on the RGDS only and combined RKR-RGDS scaffolds 436 (Figure 2). When encapsulated in hydrogels and grown in 3D, VICs retained a healthy, spread morphology (Supplemental figure S2C-D). Therefore, using a tunable scaffold with regionally localized syndecan binding (RKR) at the surface and integrin binding (RGDS) throughout the hydrogel was ideal in promoting the corresponding cell growth of VECs at the scaffold surface and VICs within (Figure 3A). The HEAVM scaffold design is the first reported direct contact co-culture model that utilizes synthetic constructs to spatially distribute multiple cell adhesive peptides in order to support layer specific cell growth as a means to mimic physiological cell organization. Previous valve cell co-culture models using collagen gels $[13,31,32]$ achieved VEC monolayer formation on the surface with VIC encapsulation underneath, but collagen gels are limited in allowing customization of specific ligand presentation and localization. The goal of the HEAVM was to build upon the work done with collagen gels by creating a synthetic scaffold with cell-specific functionalization. While specific scaffold functionalization has been

447 applied in non-contact co-culture models[14,33], the elimination of valve cell contact limits the 448 evaluation of key aspects of disease progression, such as VEC invasion or VIC disruption of the 449 endothelial layer [14,33]. Even in the broader field of vascular research, there are many examples [34] of 450 vascular endothelial cell/smooth muscle cell co-culture platforms using collagen [35], fibrin[36], 451 polytetrafluoroethylene (PTFE) [37], and poly-glycolic acid (PGA) + poly-4-hydroxybutyrate (P4HB) 452 [38] scaffold materials; none of these studies, however, incorporated spatially organized cues into the 453 scaffold designs. The HEAVM concept can also be applied to the vascular field to study atherosclerosis, 
intimal hyperplasia, cancer metastasis, and cell and tissue responses to drug models in vitro. Our model improves upon the limitations of previously reported co-culture platforms by providing customizable control of localized ligand presentation as well as mediating physiologically relevant cell localization to

457 improve the in vitro study of normal and pathological cell functions. Unlike collagen-based scaffolds,

458 this synthetic material also allows for modulation of bulk mechanical properties.

In addition to supporting cell adhesion to physiologically relevant locations within the hydrogel scaffolds, the long term maintenance of valve cell phenotypes are essential in developing a healthy aortic

461 valve model. Previous studies of valve cell co-cultures have shown to greatly influence both VIC and 462 VEC phenotypes. Specifically, the presence of VECs in co-cultures reduced VIC proliferation and $\alpha$ SMA activation, and diminished the VIC's capacity to form calcific nodules [13,15,31,32]. Conversely, a direct contact co-culture study using magnetically levitated valve cell constructs resulted in decreased VEC expression of endothelial markers (CD31, von Willebrand factor) and increased aSMA activation within the construct when surrounded by VICs[15], suggesting that localization and spatial organization of

467 VECs when co-cultured with VICs is critical for the maintenance of VEC phenotype. In HEAVM cocultures, VECs were seeded onto the surface of the scaffold, and within 7 days in culture, proliferated to form a continuous CD31 positive monolayer with limited $\alpha \mathrm{SMA}$ activation (Figure $3 \mathrm{~A}$ ), suggesting that organized placement of VECs relative to VICs on the HEAVM co-culture supported the maintenance of

471 healthy VEC phenotype (Figure 3C). Culture of VICs in 3D PEG-PQ hydrogels significantly reduced $472 \alpha$ SMA compared to 2D culture on TCPS (Figure 3B), a finding that is consistent with previously 473 published research[39]. Co-culture of VICs in the HEAVM further significantly reduced aSMA activation 474 levels in encapsulated VICs relative to VIC-only scaffolds, promoting VIC quiescence while providing an 475 organized platform for a healthy VEC layer (Figure 3B).

477 resulted in cell secretion of ECM corresponding to their location within the valve. In vivo, VECs are 478 adhered to basement membrane composed primarily of laminin, perlecan, collagen type IV, and nidogen 479 proteins [40]. Though initially supported only by RKR and RGDS ligands, VECs on the HEAVM 
constructs secreted abundant quantities of laminin, perlecan, and collagen type IV proteins localized at the

481 scaffold surface, resulting in an interwoven basement membrane to support long term endothelial growth

482 (Figure 6A). Within the scaffold, encapsulated VICs produced collagen fibers and fibronectin proteins

483 throughout the hydrogel interior, both primary components of the leaflet fibrosa layer[28,29,40],

484 suggesting the potential to produce other ECM present in mature valve tissues in future optimized

485 conditions (Figure 6B). While the simplicity of the HEAVM design provided sufficient cell adhesion

486 through the presentation of ECM-derived peptide ligands, long term co-culture of the valve cells in this

487 scaffold promoted regional production and localization of full ECM proteins.

488 Since the HEAVM design was able to maintain valve cells in stable phenotypes and promote

489 production of physiologically appropriate ECM, the model provided a platform for studies of valve cell

490 interactions and functions. Previous co-culture literature has demonstrated that VICs respond favorably to

491 co-culture with VECs, but fewer studies have focused on the VEC response. For example, NO, a

492 vasodilator, has been shown to lower VIC $\alpha$ SMA activation in vitro, and may be an important VEC

493 signaling molecule in maintaining VIC quiescence and valve tissue health [14,32]. VECs on HEAVM co-

494 culture scaffolds had similar eNOS expression, and thus, similar NO production levels in comparison to

495 VEC-only scaffolds (Figure 4B-C). Therefore, NO produced by VECs was likely a major factor in overall

496 reduction of VIC activation as seen by the lower aSMA levels of VICs in HEAVM co-cultures compared

497 to VIC-only scaffolds. In addition to influencing VIC phenotype, VECs also play a critical role in

498 regulating thrombotic and inflammatory events at the tissue surface $[11,41,42]$. In homeostatic states,

499 endothelial cells prevent platelet adhesion and activation, as depicted by the minimal platelet adhesion

500 measured and observed on VEC-only scaffolds (Figure 5A-B). There were only a very few instances of

501 platelets adhering to areas with endothelial cells on VEC-only scaffolds (Supplemental figure S5A).

502 HEAVM co-cultures promoted more platelet adhesion than the VEC-only scaffolds, but significantly less

503 than VIC-only scaffolds. Under closer inspection with confocal microscopy, the majority of the HEAVM

504 surface was covered by dil-AcLDL-positive VEC monolayers, which had minimal platelet adhesion

505 similar to VEC-only scaffolds (Figure 5E). However, platelet adhesion was visible on regions on the 
HEAVM co-culture surface that had VIC infiltration (as seen by dil-AcLDL-negative cells), blank regions

507 (lack of VEC adhesion), and disruptions in the endothelial monolayer (caused by handling of the

508 hydrogels) (Figure 5F-G, Supplemental figure S4). The HEAVM scaffold had more platelets adhered in

509 these regions than the VEC-only scaffolds because when there were breaks in the monolayer, platelets

510 adhered more readily to underlying VICs (Supplemental figure S5B) as compared to the underlying PEG-

511 PQ hydrogel in the VEC-only case (Supplemental figure S5C). Nevertheless, most of the hydrogel

512 regions with VEC monolayers reduced platelet adhesion by acting as a barrier to underlying ECM and

513 VICs, and confirms that the endothelialized hydrogel-based scaffold can maintain this essential anti-

514 thrombotic function.

515 There are still improvements that can be made in this co-culture construct design. After the

516 secondary crosslinking step in HEAVM construction, the viability of encapsulated VICs decreased. In

517 order to preserve free acrylate groups on the surface in which to bind the RKR peptide, the hydrogel was

518 initially undercrosslinked, leaving free acrylate groups both on the surface and throughout the bulk of the

519 VIC-encapsulated hydrogel. Some VICs may have been harmed during the secondary crosslinking, which

520 resulted in photoinitiated free-radicals in bulk and an increase in scaffold stiffness and decrease in pore

521 size. The surviving VICs maintained their quiescent phenotype and produced organized ECM; regardless,

522 further optimization in the HEAVM synthesis protocol will be explored to improve encapsulated cell

523 viability in future studies. Secondly, the mechanical properties and physical makeup of the current

524 HEAVM design are not representative of a physiologic valve. The base gel caused a stiff boundary

525 condition on the bottom of the co-culture gel, which VICs responded to with increased $\alpha$ SMA expression

526 (Supplemental figure S3). However, the PEGDA base gel is an improvement over stiffer substrates such

527 as glass or polystyrene. The heterogeneous ECM composition and mechanical properties of the interior of

528 the native valve leaflet are not modeled well in this system, but future work can add these properties to

529 this co-culture model. Furthermore, the HEAVM scaffold was statically cultured in this study, meaning

530 that it was not subjected to exogenously applied mechanical stimulation. Physiologically relevant

531 mechanical stimulation will likely be necessary to fully recapitulate all aspects of valve biology. 
In conclusion, we developed a novel co-culture scaffold that was used to evaluate VIC-VEC maintenance of quiescent cell phenotypes, production of ECM and NO, and prevention of platelet

534 adhesion. This system utilized the tunable characteristics of PEG-based hydrogels to localize adhesive

535 ligands shown to support cellular adhesion of specific cell types to spatially organized regions. As a

536 result, the HEAVM co-culture platforms preserved both VIC and VEC functional responses to develop a

537 simplified healthy aortic valve model. Though valve cells cultured in the HEAVM model produced

538 localized ECM within the construct, developing an aortic valve model with the valve's complex ECM

539 composition and layered structure would likely require additional approaches to recapitulate physiological

540 organization. Additional strategies such as 2-photon photo-lithography and or embedding layered

541 biomaterials into the constructs may be integrated to increase scaffold design complexity of future

542 studies[4,20]. Optimization and combinations varying the cell seeding density, adhesive ligands, and

543 growth factors can be implemented to investigate injured and diseased valve states. The application of

544 these proposed improvements to this fully synthetic scaffold could be considered in future tissue

545 engineering applications. The PEG based VIC-VEC co-culture model presented here is a next step in

546 designing a system to improve the field's understanding of how arrangement and communication between

547 VICs and VECs affect the onset and progression of valve disease.

548

549 Acknowledgements

550 This research was funded by a predoctoral fellowship from the National Science Foundation (to

551 D.S.P), a predoctoral fellowship from the American Heart Association (to L.R.B), and a grant from the

552 National Institutes of Health (R01HL107765). The authors thank Dr. Zarana Patel for fruitful initial

553 discussions and the Drezek Lab at Rice University for kindly sharing equipment. 


\section{References}

557 [1] Baker BM, Chen CS. Deconstructing the third dimension: how 3D culture microenvironments alter cellular cues. J Cell Sci 2012;125:3015-24. doi:10.1242/jcs.079509.

559

560

561

562

563

564

565

566

567

568

569

570

571

572

573

574

575

576

577

578

579

580

581

582

583

584

585

586

587

588

589

590
[2] Hockaday LA, Kang KH, Colangelo NW, Cheung PYC, Duan B, Malone E, et al. Rapid 3D printing of anatomically accurate and mechanically heterogeneous aortic valve hydrogel scaffolds. Biofabrication 2012;4:035005. doi:10.1088/1758-5082/4/3/035005.

[3] Malda J, Visser J, Melchels FP, Jüngst T, Hennink WE, Dhert WJ a, et al. 25th anniversary article: Engineering hydrogels for biofabrication. Adv Mater 2013;25:5011-28.

doi:10.1002/adma.201302042.

[4] Hoffmann JC, West JL. Three-dimensional photolithographic patterning of multiple bioactive ligands in poly(ethylene glycol) hydrogels. Soft Matter 2010;6:5056. doi:10.1039/c0sm00140f.

[5] Lee W, Debasitis JC, Lee VK, Lee J-H, Fischer K, Edminster K, et al. Multi-layered culture of human skin fibroblasts and keratinocytes through three-dimensional freeform fabrication. Biomaterials 2009;30:1587-95. doi:10.1016/j.biomaterials.2008.12.009.

[6] Tsuda Y, Shimizu T, Yamato M, Kikuchi A, Sasagawa T, Sekiya S, et al. Cellular control of tissue architectures using a three-dimensional tissue fabrication technique. Biomaterials 2007;28:493946. doi:10.1016/j.biomaterials.2007.08.002.

[7] Battiston KG, Cheung JWC, Jain D, Santerre JP. Biomaterials in co-culture systems: towards optimizing tissue integration and cell signaling within scaffolds. Biomaterials 2014;35:4465-76. doi:10.1016/j.biomaterials.2014.02.023.

[8] Chwalek K, Tsurkan M V, Freudenberg U, Werner C. Glycosaminoglycan-based hydrogels to modulate heterocellular communication in in vitro angiogenesis models. Sci Rep 2014;4:4414. doi:10.1038/srep04414.

[9] L Berg E, Hsu Y-C, Lee J a. Consideration of the cellular microenvironment: physiologically relevant co-culture systems in drug discovery. Adv Drug Deliv Rev 2014;69-70:190-204. doi:10.1016/j.addr.2014.01.013.

[10] Taylor PM, Batten P, Brand NJ, Thomas PS, Yacoub MH. The cardiac valve interstitial cell. Int J Biochem Cell Biol 2003;35:113-8.

[11] Balaoing LR, Post AD, Liu H, Minn KT, Grande-Allen KJ. Age-related changes in aortic valve hemostatic protein regulation. Arterioscler Thromb Vasc Biol 2014;34:72-80. doi:10.1161/ATVBAHA.113.301936.

[12] Leask RL, Jain N, Butany J. Endothelium and valvular diseases of the heart. Microsc Res Tech 2003;60:129-37. doi:10.1002/jemt.10251.

[13] Butcher JT, Nerem RM. Valvular endothelial cells regulate the phenotype of interstitial cells in coculture: effects of steady shear stress. Tissue Eng 2006;12:905-15. doi:10.1089/ten.2006.12.905. 
[14] Gould ST, Matherly EE, Smith JN, Heistad DD, Anseth KS. The role of valvular endothelial cell paracrine signaling and matrix elasticity on valvular interstitial cell activation. Biomaterials 2014;35:3596-606. doi:10.1016/j.biomaterials.2014.01.005.

[15] Tseng H, Balaoing LR, Grigoryan B, Raphael RM, Killian TC, Souza GR, et al. A threedimensional co-culture model of the aortic valve using magnetic levitation. Acta Biomater 2014;10:173-82. doi:10.1016/j.actbio.2013.09.003.

[16] Stephens EH, Carroll JL, Grande-Allen KJ. The use of collagenase III for the isolation of porcine aortic valvular interstitial cells: rationale and optimization. J Heart Valve Dis 2007;16:175-83.

[17] Cheung W-Y, Young EWK, Simmons $\mathrm{C}$ a. Techniques for isolating and purifying porcine aortic valve endothelial cells. J Heart Valve Dis 2008;17:674-81.

[18] Butcher JT, Penrod AM, García AJ, Nerem RM. Unique morphology and focal adhesion development of valvular endothelial cells in static and fluid flow environments. Arterioscler Thromb Vasc Biol 2004;24:1429-34. doi:10.1161/01.ATV.0000130462.50769.5a.

[19] Moon JJ, Saik JE, Poché R a, Leslie-Barbick JE, Lee S-H, Smith A a, et al. Biomimetic hydrogels with pro-angiogenic properties. Biomaterials 2010;31:3840-7. doi:10.1016/j.biomaterials.2010.01.104.

[20] Tseng H, Puperi DS, Kim EJ, Ayoub S, Shah J V, Cuchiara ML, et al. Anisotropic Poly(Ethylene Glycol)/Polycaprolactone Hydrogel-Fiber Composites for Heart Valve Tissue Engineering. Tissue Eng Part A 2014;00:1-12. doi:10.1089/ten.TEA.2013.0397.

[21] Bahney CS, Lujan TJ, Hsu CW, Bottlang M, West JL, Johnstone B. Visible light photoinitiation of mesenchymal stem cell-laden bioresponsive hydrogels. Eur Cell Mater 2011;22:43-55; discussion 55 .

[22] Pfaffl MW, Horgan GW, Dempfle L. Relative expression software tool (REST) for group-wise comparison and statistical analysis of relative expression results in real-time PCR. Nucleic Acids Res 2002;30:e36.

[23] Xu X-X, Gao X-H, Pan R, Lu D, Dai Y. A simple adhesion assay for studying interactions between platelets and endothelial cells in vitro. Cytotechnology 2010;62:17-22. doi:10.1007/s10616-010-9256-2.

[24] Peppas NA, Hilt JZ, Khademhosseini A, Langer R. Hydrogels in Biology and Medicine: From Molecular Principles to Bionanotechnology. Adv Mater 2006;18:1345-60. doi:10.1002/adma.200501612.

[25] Benton JA, Fairbanks BD, Anseth KS. Characterization of valvular interstitial cell function in three dimensional matrix metalloproteinase degradable PEG hydrogels. Biomaterials 2009;30:6593-603. doi:10.1016/j.biomaterials.2009.08.031.

[26] Gould ST, Anseth KS. Role of cell-matrix interactions on VIC phenotype and tissue deposition in 3D PEG hydrogels. J Tissue Eng Regen Med 2013. doi:10.1002/term.1836. 
[27] Balaoing LR, Post AD, Lin AY, Tseng H, Moake JL, Grande-Allen KJ. Laminin PeptideImmobilized Hydrogels Modulate Valve Endothelial Cell Hemostatic Regulation. PLoS One 2015;10:e0130749. doi:10.1371/journal.pone.0130749.

[28] Latif N, Sarathchandra P, Taylor PM, Antoniw J, Yacoub MH. Localization and pattern of expression of extracellular matrix components in human heart valves. J Heart Valve Dis 2005;14:218-27.

[29] Hinton RB, Yutzey KE. Heart valve structure and function in development and disease. Annu Rev Physiol 2011;73:29-46. doi:10.1146/annurev-physiol-012110-142145.

[30] Morgan MR, Humphries MJ, Bass MD. Synergistic control of cell adhesion by integrins and syndecans. Nat Rev Mol Cell Biol 2007;8:957-69. doi:10.1038/nrm2289.

[31] Flanagan TC, Wilkins B, Black A, Jockenhoevel S, Smith TJ, Pandit AS. A collagenglycosaminoglycan co-culture model for heart valve tissue engineering applications. Biomaterials 2006;27:2233-46. doi:10.1016/j.biomaterials.2005.10.031.

[32] Richards J, El-Hamamsy I, Chen S, Sarang Z, Sarathchandra P, Yacoub MH, et al. Side-specific endothelial-dependent regulation of aortic valve calcification: interplay of hemodynamics and nitric oxide signaling. Am J Pathol 2013;182:1922-31. doi:10.1016/j.ajpath.2013.01.037.

[33] Chen MB, Srigunapalan S, Wheeler AR, Simmons C a. A 3D microfluidic platform incorporating methacrylated gelatin hydrogels to study physiological cardiovascular cell-cell interactions. Lab Chip 2013;13:2591-8. doi:10.1039/c3lc00051f.

[34] Ravi S, Chaikof EL. Biomaterials for vascular tissue engineering. Regen Med 2010;5:107-20. doi:10.2217/rme.09.77.

[35] Weinberg CB, Bell E. A blood vessel model constructed from collagen and cultured vascular cells. Science 1986;231:397-400.

[36] Swartz DD, Russell JA, Andreadis ST. Engineering of fibrin-based functional and implantable small-diameter blood vessels. Am J Physiol Heart Circ Physiol 2005;288:H1451-60. doi:10.1152/ajpheart.00479.2004.

[37] Chen L, Yu H, Dai N, Tao S, Gong W. [Dual cell seeding to improve cell retention on polytetrafluoroethylene grafts]. Zhonghua Wai Ke Za Zhi 2003;41:143-5.

[38] Hoerstrup SP, Cummings Mrcs I, Lachat M, Schoen FJ, Jenni R, Leschka S, et al. Functional growth in tissue-engineered living, vascular grafts: follow-up at 100 weeks in a large animal model. Circulation 2006;114:I159-66. doi:10.1161/CIRCULATIONAHA.105.001172.

[39] Wang H, Tibbitt MW, Langer SJ, Leinwand L a, Anseth KS. Hydrogels preserve native phenotypes of valvular fibroblasts through an elasticity-regulated PI3K/AKT pathway. Proc Natl Acad Sci U S A 2013;110:19336-41. doi:10.1073/pnas.1306369110.

[40] Wiltz D, Arevalos CA, Balaoing LR, Blancas AA, Sapp MC, Zhang X, et al. Calcific Aortic Valve Disease. In: Aikawa E, editor., InTech; 2013, p. 3-30. doi:10.5772/46239. 
663 [41] Leask RL, Jain N, Butany J. Endothelium and valvular diseases of the heart. Microsc Res Tech $664 \quad 2003 ; 60: 129-37$. doi:10.1002/jemt.10251.

665 [42] Hakuno D, Kimura N, Yoshioka M, Fukuda K. Molecular mechanisms underlying the onset of 666 degenerative aortic valve disease. J Mol Med 2009;87:17-24. doi:10.1007/s00109-008-0400-9.

667

668 


\section{Figure Captions}

670

671

672

673

674

675

676

677

678

679

680

681

682

683

684

685

686

687

688

689

690

691

692

Figure 1. (A) Schematic of hydrogel-based endothelialized aortic valve model (HEAVM) co-culture construction. (B) HEAVM scaffold on base gel. (C) Fluorescently tagged RKR+AF532 (red) on the surface of RKR conjugated scaffold (left only). PEG-RGDS+AF488 (green) present throughout the hydrogel. Scale bars $=100 \mu \mathrm{m}$

Figure 2. Aortic endothelial cells (AECs), valve endothelial cells (VECs) and valve interstitial cells (VICs) cultured on the surface of PEG-PQ scaffolds immobilized with +RGDS/-RKR, -RGDS/+RKR, or + RKR/+RGDS. (A) Brightfield images show that cell adhesion and proliferation are affected by adhesive ligand combination. Black scale bars $=100 \mu \mathrm{m}$. (B) Confluent regions of VECs and AECs stained for CD31 (green) and no $\alpha$ SMA (red) on all scaffold combinations. VICs stained for $\alpha$ SMA on all scaffold combinations, but had limited spreading on the -RGDS/+RKR scaffold. White scale bars $=50 \mu \mathrm{m}$

Figure 3. (A) After 7 days in culture, HEAVM co-cultures maintain valve cell phenotypes. VECs on the surface of the gel formed a confluent monolayer and express CD31 (green), while encapsulated cells had low levels of $\alpha$ SMA (scale bars $=50 \mu \mathrm{m}$ ). (B) HEAVM co-cultures, VEC only scaffolds, and VECs on TCPS all express significantly less $\alpha$ SMA mRNA relative to VIC only scaffolds. VICs cultured on TCPS express significantly more $\alpha$ SMA mRNA than all other groups. (C) All VEC based cultures have similar levels of CD31 gene expression, while VIC only scaffolds express significantly less CD31 than all other groups. ${ }^{*} p<0.05$ versus all others. ${ }^{\wedge} \mathrm{p}<0.05$ versus all others. $\mathrm{n}=4$

Figure 4. (A) HEAVM co-cultures stain for eNOS (magenta) co-localized with CD31 positive (green) VECs at the surface of the scaffold (Scale bars $=50 \mu \mathrm{m}$ ). (B) HEAVM co-cultures had similar levels of eNOS gene expression as VEC only scaffolds and VECs on TCPS, and all had significantly higher eNOS gene expression than VIC only scaffolds. $n=4$ (C) VEC only and HEAVM scaffolds released significantly higher concentrations of nitric oxide byproducts (that were converted into nitrates) into the culture medium than VIC only scaffolds. $\mathrm{n}=5 .{ }^{*} p<0.05$ between noted samples 
Figure 5. (A) Adhered platelets on VEC only, VIC only, and HEAVM scaffolds relative to the number of

694 adhered platelets on blank acellular scaffolds. HEAVM co-cultures had significantly less platelet

695 adhesion than VIC only scaffolds, but significantly more adhered platelets than VEC only cultures. VIC

696 only was significantly higher than all other cases. HEAVM was significantly greater than VEC only, but

697 significantly less than VIC only, but not significantly different from blank gel, $n=6-8$. Bars that are not

698 connected be the same letter significantly different. $p<0.05$. (B) VEC only scaffolds stained for Dil-

699 AcLDL and had minimal visible platelet adhesion across the hydrogel surface. (C). Large platelet clumps

700 adhered to regions where VICs were present on the surface on VIC only scaffolds. (D) Platelets adhered

701 on acellular, blank HEAVM scaffolds. (E) Most HEAVM co-culture scaffolds stained for Dil-AcLDL on

702 the surface coated monolayer and had minimal platelet adhesion. (F) Blank regions and $(\mathrm{G})$ cells negative

703 to Dil-AcLDL uptake on HEAVM co-cultures promoted platelet clump adhesion. Scale bars $=50 \mu \mathrm{m}$

704 Figure 6. (A). HEAVM co-cultures produce basement membrane components laminin (red), perlecan

705 (green), and Col IV (purple) at the surface of the scaffold within 28 days of culture. (B) Fibronectin (FN)

706 (red) is present throughout the surface (top) and within the HEAVM scaffold, while Col I fibers (green)

707 are present beginning 10um below the scaffold surface (bottom). Scale bars $=50 \mu \mathrm{m}$ 
A. VICs in PEG-PQ-PEG +
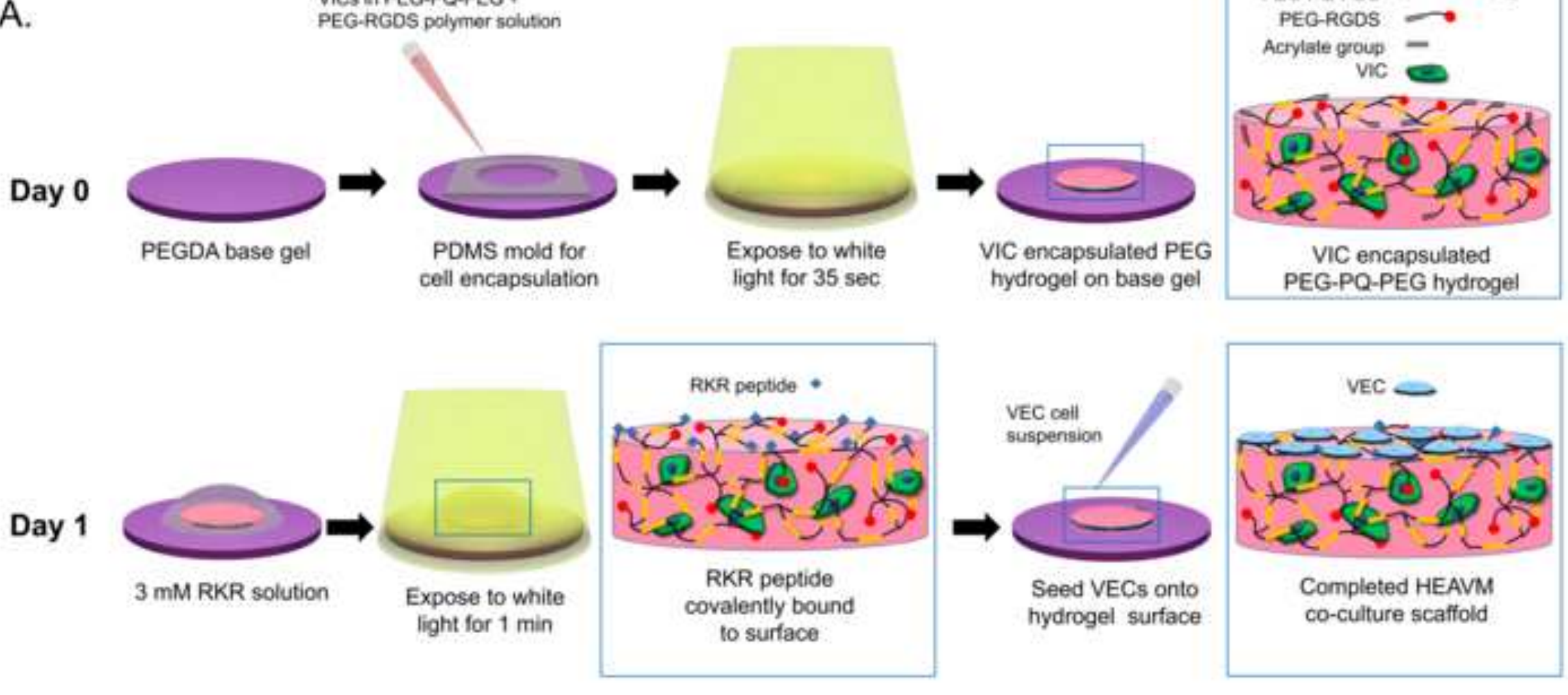

B.

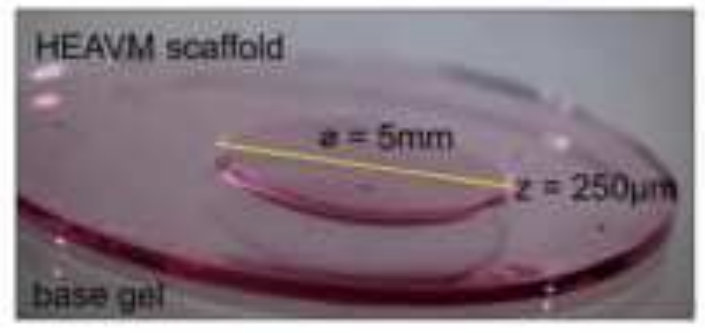

C.

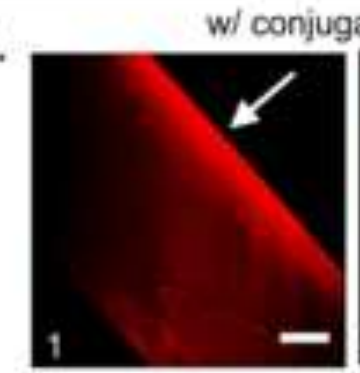

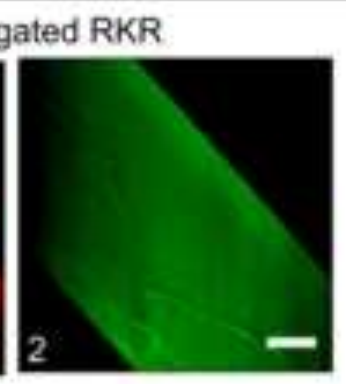

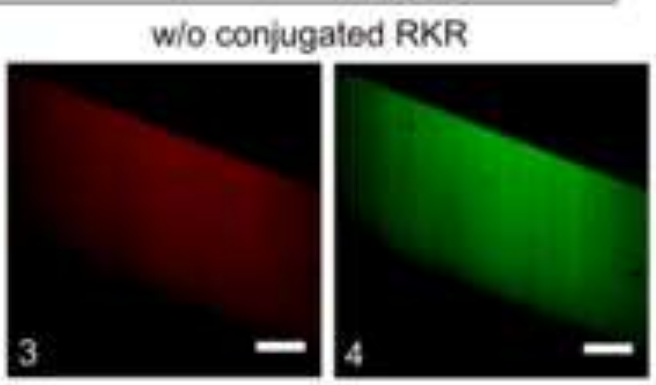



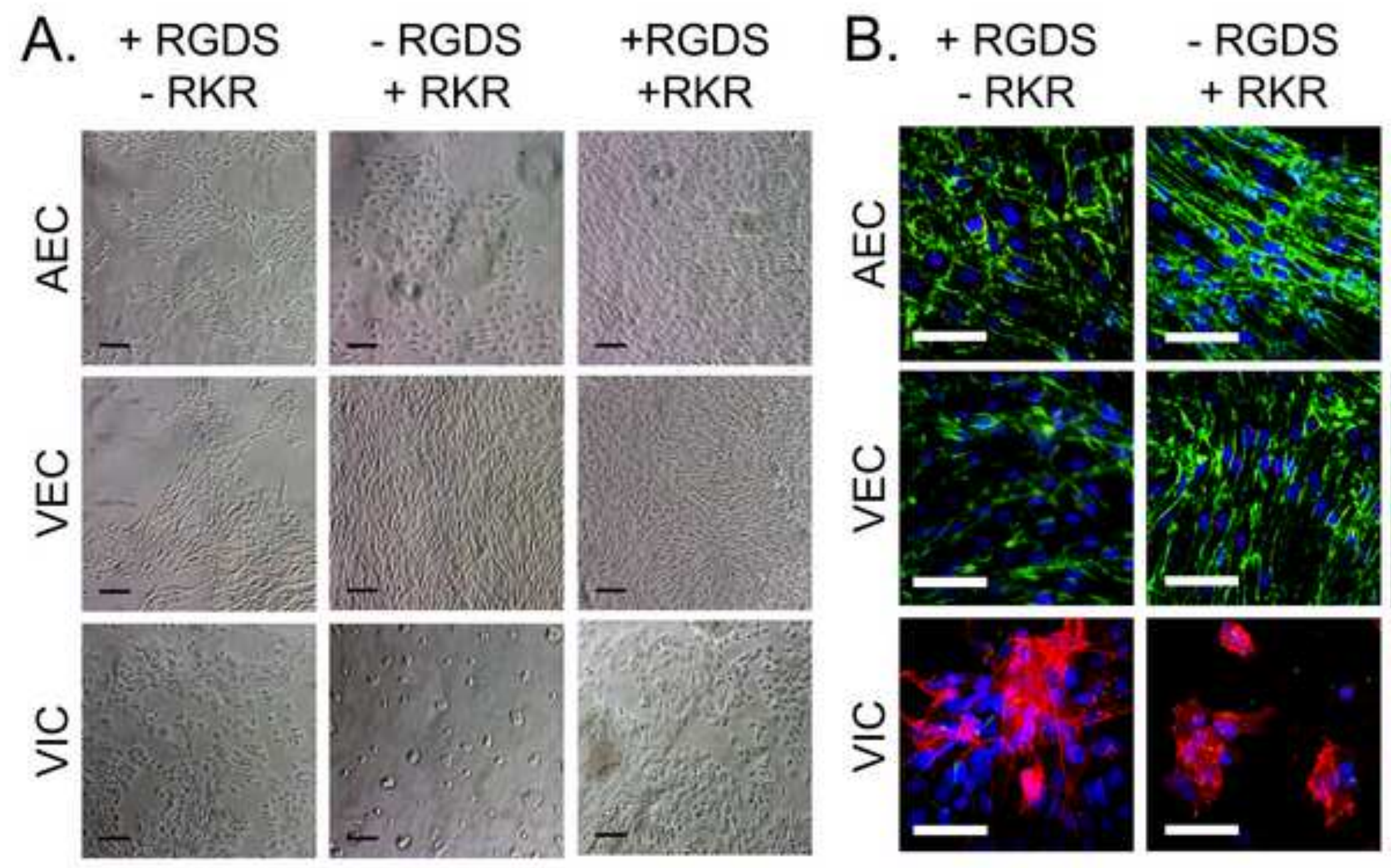

+RGDS + RKR
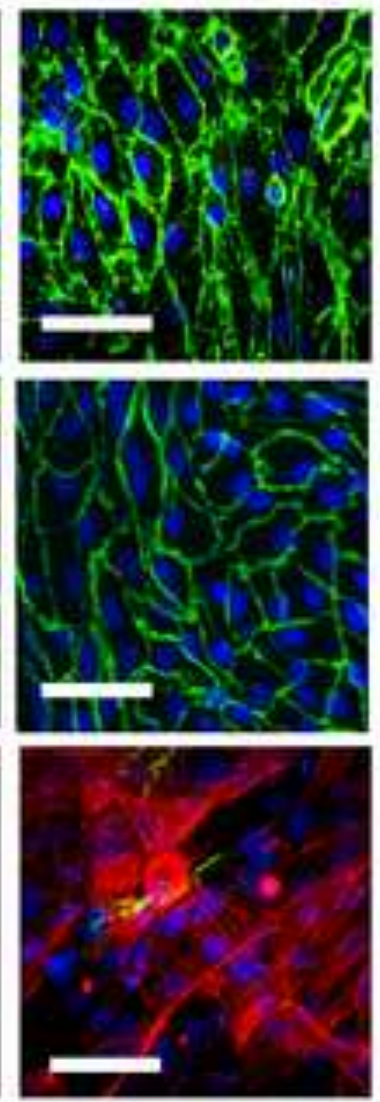

A. + RGDS

$\underset{1}{3}$
RGDS 

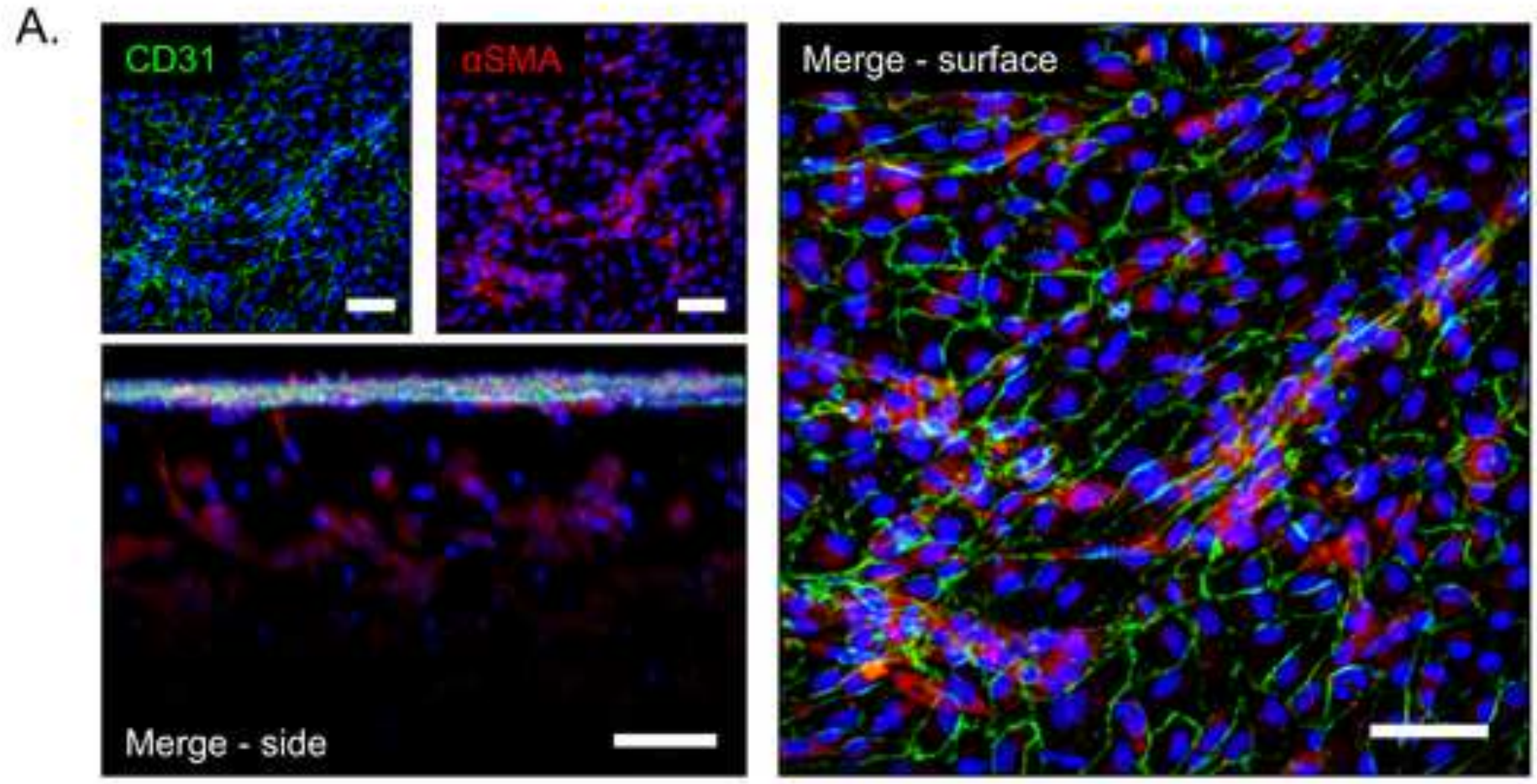

B.

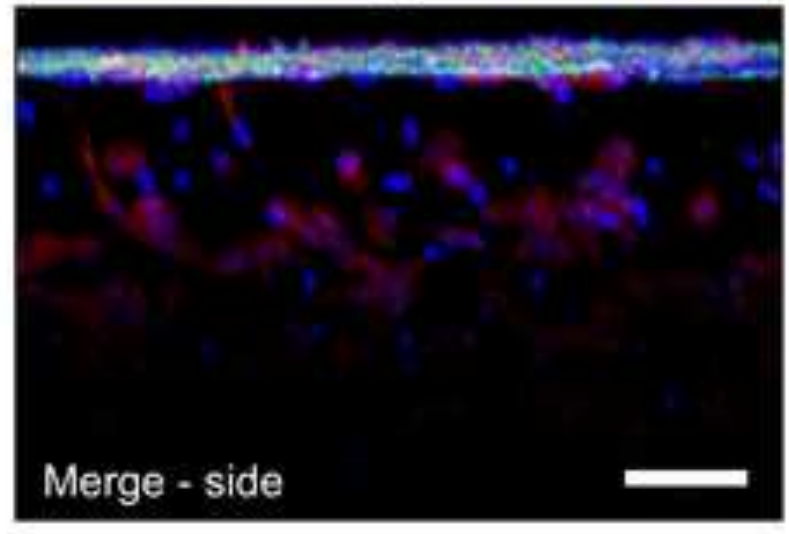

C.
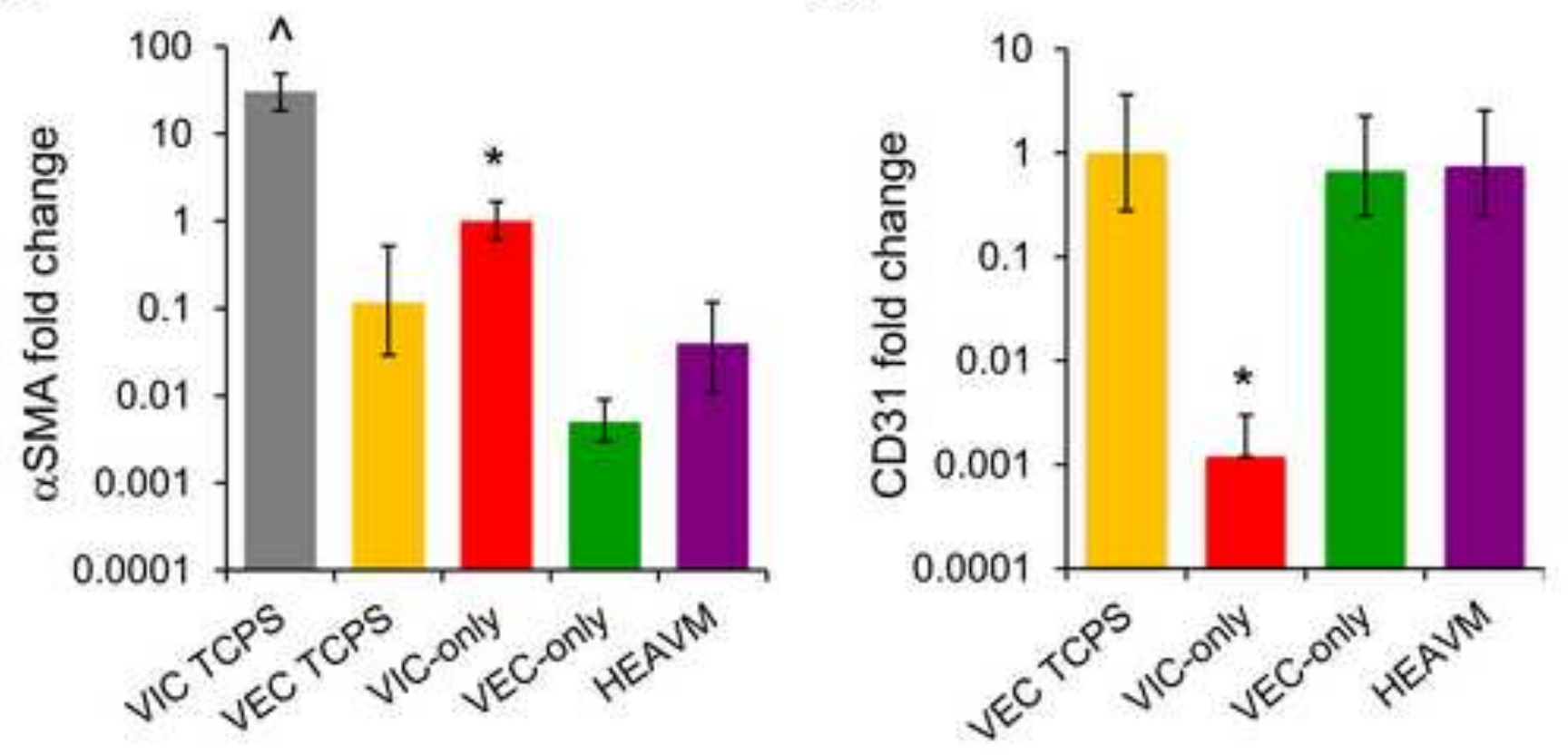
Figure 4
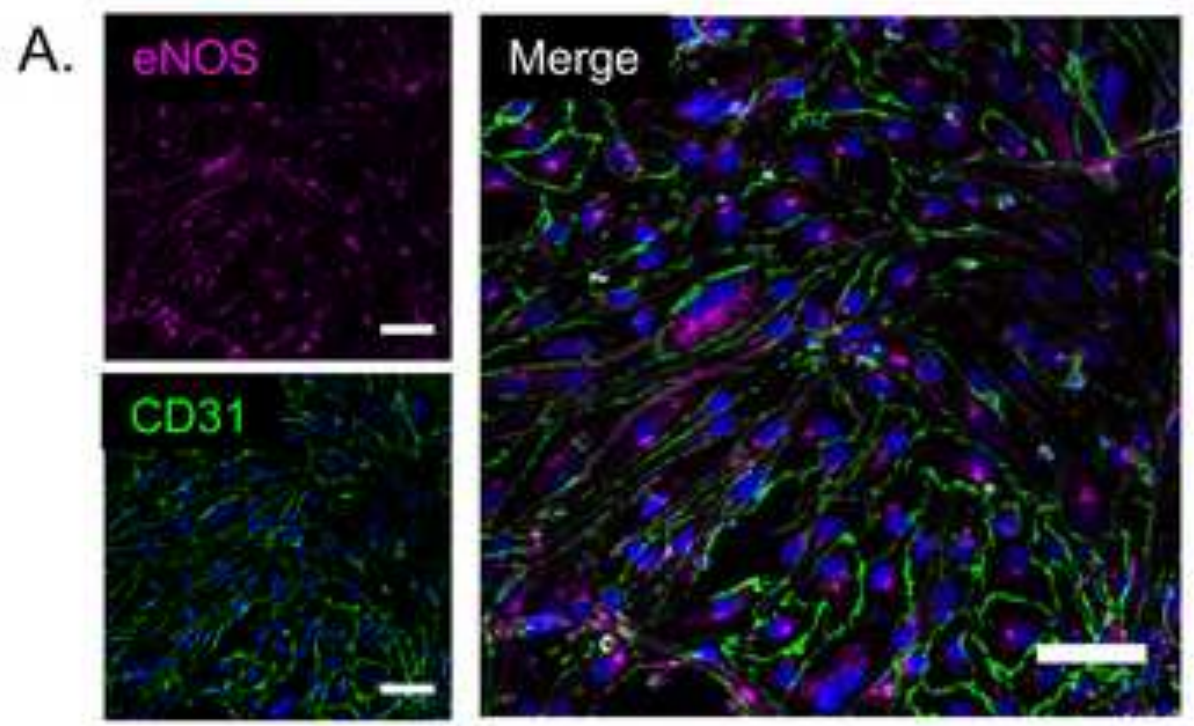

B.

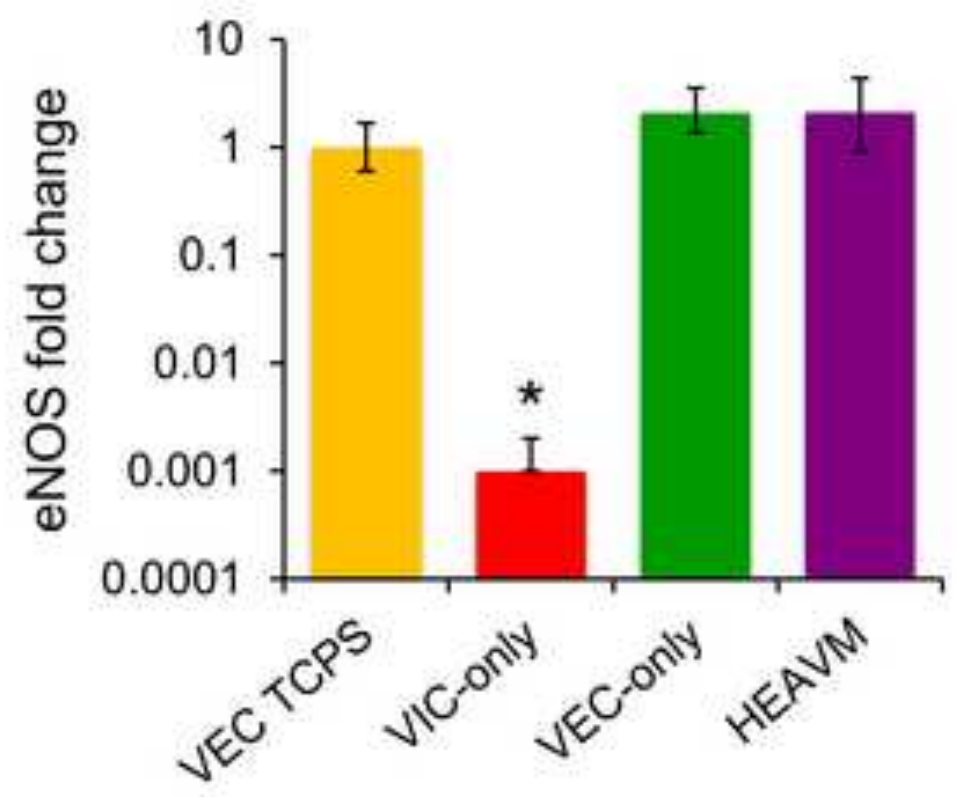

C.

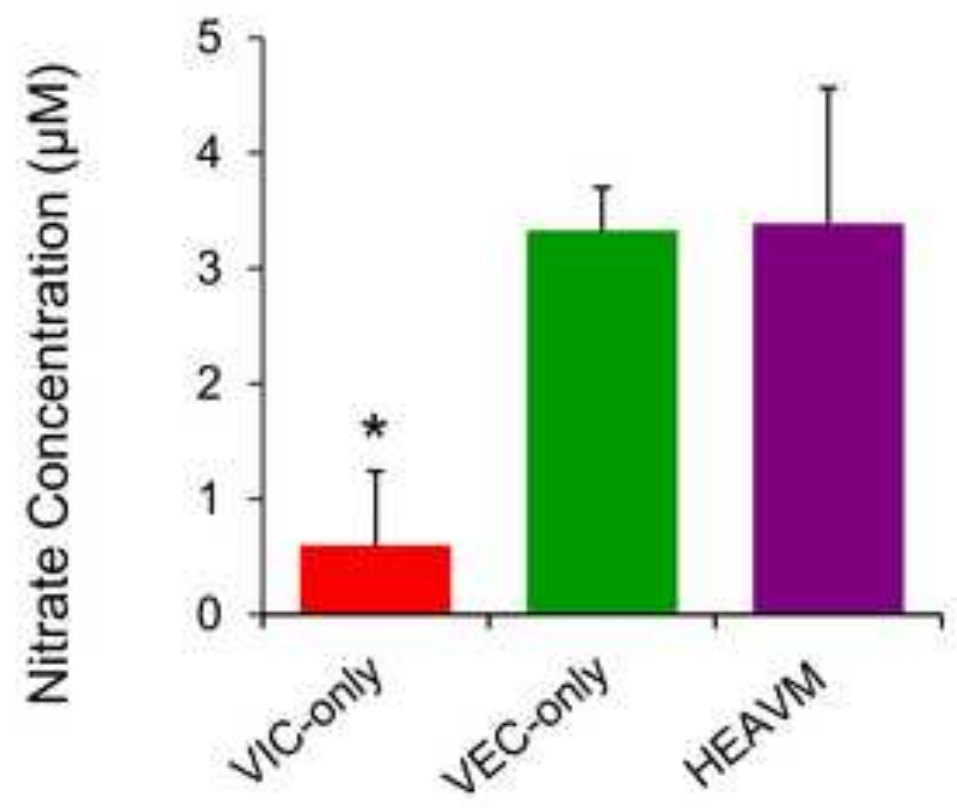



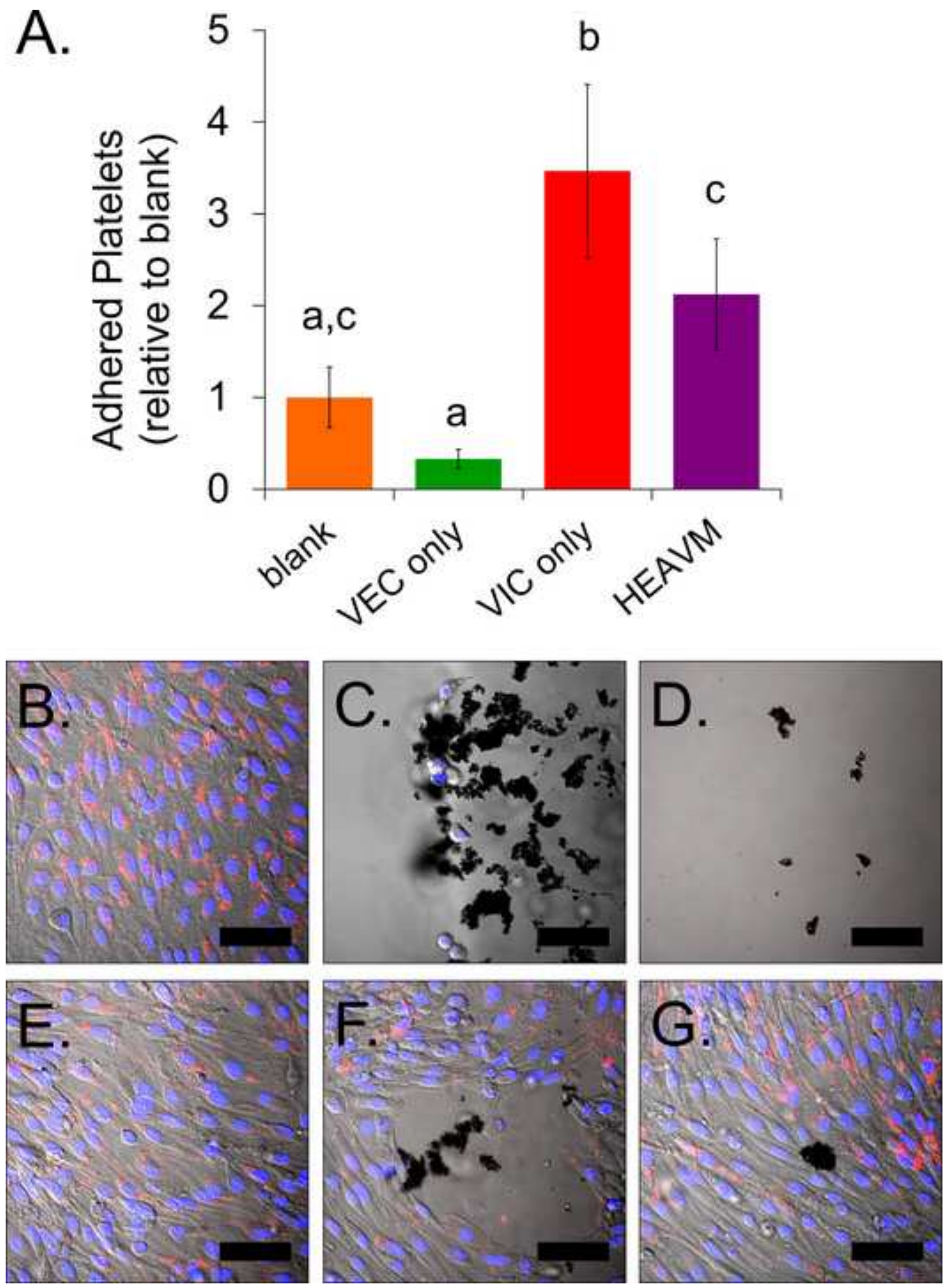

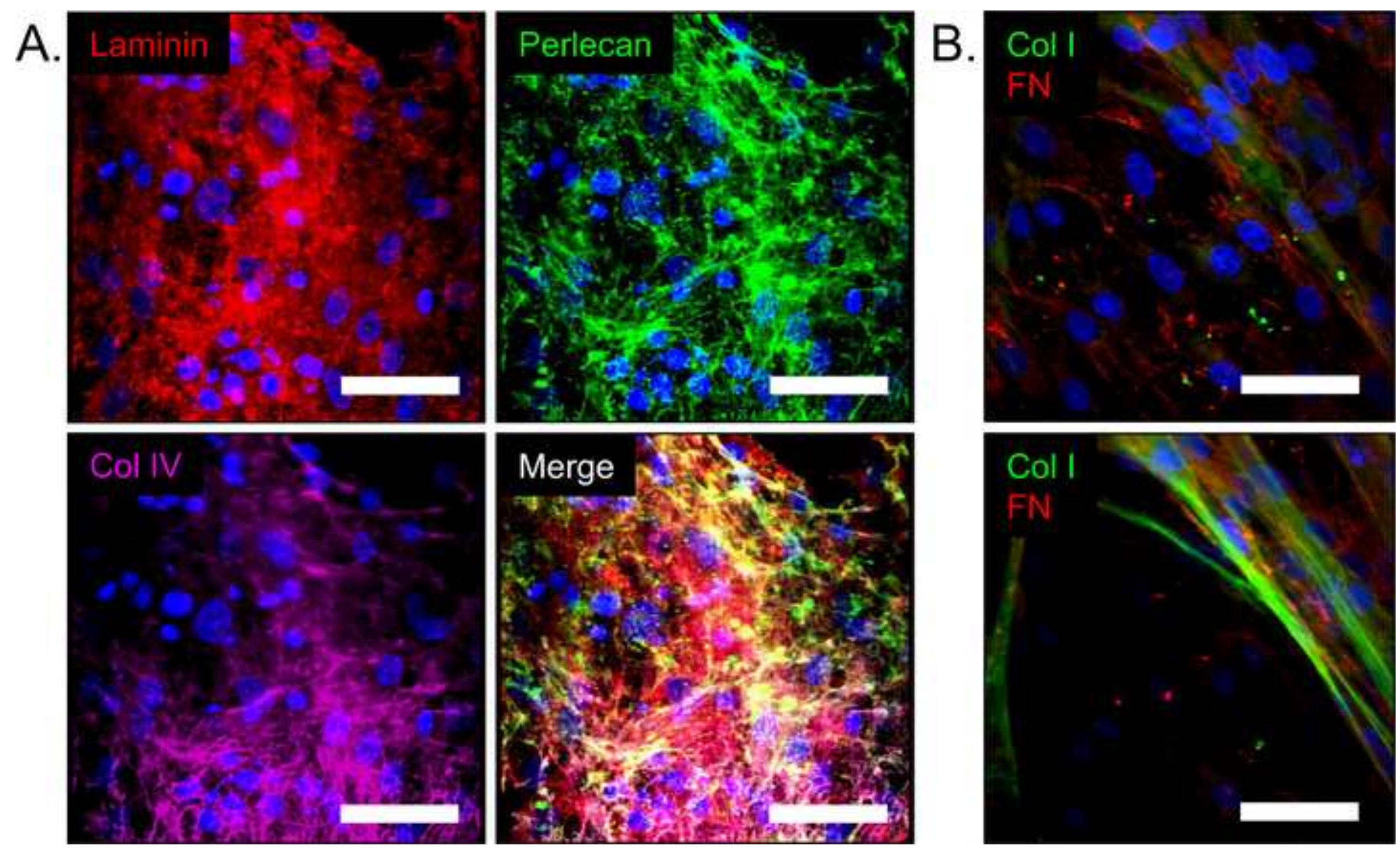